\title{
Coherencia curricular y oportunidades para aprender Ciencias*
}

\section{Curriculum coherence and opportunities to learn Science}

\author{
Marcela Ruiz Zúñiga ${ }^{1}$ • Alejandra Meneses Arévalo ${ }^{2}$ • \\ Maximiliano Montenegro Maggio ${ }^{2}$
}

\begin{abstract}
Resumen: Se determinó la coherencia curricular entre oportunidades de aprendizaje y habilidades científicas ofrecidas por un texto escolar chileno y por las actividades de aula en $5^{\circ}$ grado asociadas al contenido "Fuerza". Se realizó un análisis discursivo de la unidad didáctica del texto y de las actividades con el fin de construir matrices de contenido para tópicos y habilidades; la medición de coherencia curricular se realizó a través del índice de Porter para la coherencia. Los resultados muestran que el texto escolar promueve más observación $(24.2 \%)$ y menos ordenamiento de la información $(0.95 \%)$. En las clases, predominó repetición de contenidos de manera oral y escrita $(66.8 \%)$ y menos análisis de hipótesis (1.6\%). El índice de Porter entre el texto y el aula fue 0.360 , entre el texto y el marco curricular fue 0.267 . Se concluye que el texto y las clases ofrecen leves oportunidades para el desarrollo de las habilidades científicas.
\end{abstract}

Palabras clave: Aprendizaje. Habilidad científica. Coherencia curricular. Alfabetización científica.

\begin{abstract}
We obtain curriculum coherence between opportunities to learn scientific skills offered in a Chilean textbook and those offered in the classroom about the concept of force in a fifth grade classroom. We performed a discourse analysis of the lesson unit and the classroom activities to obtain content matrices for the concepts involved and the scientific skills promoted. To measure the curricular coherence we used the Porter index. Our results show that the textbook greatly promotes observation $(24.2 \%)$, whereas data management is the least promoted $(0.95 \%)$. In the classroom, content presentation predominates $(66.8 \%)$, whereas hypothesis analysis is the less favored $(1.6 \%)$. The Porter index obtained between the textbook and the classroom activities was 0.360 , whereas between the textbook and the national curricula was 0.267 . Our results show that both the textbook and the classroom activities offer low opportunities to develop scientific skills to the students.
\end{abstract}

Keywords: Learning. Scientific skills. Curricular coherence. Scientific literacy.

\footnotetext{
*Investigación financiada por FONDECYT 1130953.

${ }^{1}$ Departamento de Lengua y Literatura, Facultad de Filosofía y Humanidades, Universidad Alberto Hurtado (UAH), Santiago, Región Metropolitana, Chile. Email: <maruiz@uahurtado.cl>

${ }^{2}$ Departamento de Didáctica, Facultad de Educación, Pontificia Universidad Católica de Chile (PUC), Santiago, Chile.
} 


\section{Introducción}

La preocupación por las metas y concepciones de la enseñanza de las ciencias en la escuela se ha incrementado en varios países occidentales (Inglaterra, Noruega, Dinamarca, Finlandia, Estados Unidos, Francia, entre otros), debido al imperativo de competir en una economía globalizada frente a la pujante innovación científico-tecnológica liderada por China e India. A esta tendencia se suman las declaraciones de organismos internacionales (UNESCO, Organización para la Cooperación y el Desarrollo Económicos, Banco Mundial) que reconocen que su incorporación curricular es una pieza relevante para el desarrollo económico. (DILLON, 2009; JENKIS, 2009; LAVONEN; LAAKSONEN, 2009; MILLAR, 2006).

Esta preocupación ha puesto de relieve la alfabetización cientifica a la hora de determinar las orientaciones curriculares nacionales. Aun cuando su empleo está ampliamente difundido desde 1950, su significado y alcance es objetode constante discusión bibliográfica, de modo que no existe una definición única (DEBOER, 2000; DILLON, 2009; FENSHAM, 2002; HOLBROOK; RANNIKMAE, 2009; HURD,1998; LAUGKSCH, 2000). Sin embargo, es posible distinguir ciertos aspectos centrales relacionados con el dominio de los conocimientos y procesos científicos en la escuela como la naturaleza e historia del saber científico y el aprendizaje de habilidades como formulación de hipótesis, experimentación, construcción de modelos, uso de evidencias, entre otros. (FENSHAM, 2002; LIU, 2009; ROBERTS, 2007).

Tal como se indicó, la alfabetización científica es un concepto que cada currículum nacional re-significa. Por lo tanto, es necesario precisar de qué manera es formulado en el currículo nacional chileno. (CHILE, 2010). La actualización curricular (CHILE, 2008) para el subsector de Ciencias Naturales reorganiza y amplía los ejes de los contenidos científicos de cuatro a cinco, a saber: Estructura y función de los seres vivos, Organismos, ambiente y sus interacciones, La materia y sus transformaciones, Fuerza y Movimiento, La Tierra y el Universo. explicitan habilidades científicas denominadas en el currículo oficial como habilidades de pensamiento científico que constituyen un eje independiente y transversal. Esta modificación apunta a destacar el lugar de las capacidades propias de las habilidades científicas con respecto al aprendizaje de los contenidos. Si bien estas ya estaban presentes en la versión previa del Marco Curricular (CHILE, 2002), carecían de una presencia notoria para este subsector de aprendizaje (CHILE, 2009b). De esta manera, se logra una mayor sintonía con las metas propias de la alfabetización científica requeridas por el contexto de desarrollo mundial.

En primer lugar, el currículo chileno del 2009 declara que el propósito de la enseñanza de las Ciencias consiste en el desarrollo de "[...] la capacidad de usar el conocimiento científico, de identificar problemas y de esbozar conclusiones basadas en evidencia, en orden a entender y participar de las decisiones sobre el mundo natural y los cambios provocados por la actividad humana" (CHILE, 2010, p. 243).

En este planteamiento, por un lado, son destacadas las habilidades de razonamiento y el procedimiento experimental - "la formulación de preguntas, la observación, la descripción y registro de datos, el ordenamiento e interpretación de información, la elaboración y el análisis de hipótesis, los procedimientos y explicaciones, la argumentación y el debate" (CHILE, 2010, p. 245) - que deben estar presentes en cada eje de conocimiento científico. Por otro lado, la argumentación y el debate se desarrollan en contextos socio comunicativos, donde se enfrentan y evalúan conclusiones y sus justificaciones/refutaciones, sustentadas en la organización e 
interpretación de la evidencia. Las consecuencias del debate pueden trascender el contenido disciplinar específico y expandirse al ámbito social -“en torno a controversias y problemas de interés público, y a la discusión y evaluación de implicancias éticas o ambientales relacionadas con la ciencia y la tecnología" (CHILE, 2010, p. 245).

De este modo, se observa que el aprendizaje de los conceptos científicos no tiene una finalidad en sí mismo, sino que el foco es el desarrollo de las habilidades científicas asociadas a diferentes conceptos científicos y contextos socio-científicos. Estos contextos se refieren a fenómenos del mundo natural y a las modificaciones causadas por el ser humano. Si bien en la definición no está explicitado su tipo, posteriormente, se señala que corresponden a instancias de la vida de los estudiantes, específicamente, a ámbitos sociales relacionados con la calidad de vida y la protección del entorno, en particular, medio ambiente, tecnología y salud (CHILE, 2010). Así, se prepara a los estudiantes para los desafíos propios del aprendizaje continuo de la sociedad de la información mediante el desarrollo de las capacidades de uso crítico de la información sobre los avances científicos.

En síntesis, la alfabetización científica en el marco curricular chileno del 2009 (CHILE, 2010) pone de relieve las habilidades vinculadas con los contenidos de los ejes temáticos. Estas habilidades incluyen las operaciones distintivas de la investigación empírica, pero no están adscritas a una secuencia específica. También se consideran otras relacionadas con las formas de hacer y comunicar ciencias, tales como la argumentación y el debate sobre cuestiones de relevancia pública y ética. Es importante advertir que tales habilidades y conocimientos deben utilizarse en contextos experienciales de los estudiantes.

Si bien ha habido una prescripción curricular que ha ido explicitando paulatinamente la importancia del desarrollo de las habilidades científicas, Chile ha mostrado - en esta última década - un bajo desempeño en ciencias. Los resultados de la prueba Trends in International Mathematics and Science Study (TIMSS) 2003 muestran que un 44\% de los estudiantes chilenos de $8^{\circ}$ básico alcanzan el nivel de logro inferior, un $19 \%$ el logro bajo y solo un $1 \%$ el nivel avanzado.

En efecto, el rendimiento de los estudiantes chilenos es considerablemente menor a los resultados obtenidos por países como Estados Unidos, Hong Kong, Australia y Malasia (CHILE, 2004). Los contenidos curriculares menos logrados corresponden al área de física y solo un $1 \%$ de los estudiantes desarrolla explicaciones escritas sobre conceptos científicos complejos y abstractos para las cuales se requieren habilidades de razonamiento y análisis.

Por otro lado, la prueba PISA 2006 muestra que 69,7\% de los estudiantes chilenos se encuentran en los tres niveles más bajo de desempeño; en cambio, solo un 5,2\% de los países desarrollados se ubican en dichos niveles. PISA 2006 revela que solo un 1,8\% de los estudiantes chilenos es capaz de resolver problemas científicos demostrando un razonamiento avanzado y utilizando evidencias provenientes de diversas fuentes de información (CHILE, 2007). El análisis estadístico para la conformación de conglomerados realizado por Gubler y Williamson (2009) identificó en la subescala "usar evidencia científica" que las habilidades menos desarrolladas son interpretar pruebas científicas y formular conclusiones. Por su parte, para la subescala "identificar cuestiones científicas", la habilidad menos desarrollada es reconocer cuestiones susceptibles de ser investigadas científicamente. Dicho estudio concluyó que "estos resultados muestran la necesidad de enfatizar y fortalecer más en Chile el desarrollo de habilidades de pensamiento científico entre los estudiantes." (GUBLER; WILLIAMSON, 2009, p. 224). 
Por lo tanto, como muestran distintas pruebas estandarizadas internacionales, los resultados de aprendizaje de los estudiantes chilenos son bastante deficientes en el dominio de contenidos, así como en el desarrollo de las habilidades científicas. Aún cuando el sistema educativo chileno ha sufrido, desde hace más de 15 años, importantes cambios curriculares, incentivos a los docentes, inversiones en infraestructuras escolares, desarrollo de una política pública de edición y entrega de textos escolares, dichas políticas públicas no se han visto reflejadas aún en el aumento de los aprendizajes de los estudiantes.

Considerando, entonces, los bajos desempeños en el área de Ciencia y el continuo contexto de ajuste curricular, es relevante indagar en torno a las habilidades científicas que son efectivamente promovidas en el sistema escolar chileno. Específicamente, se busca determinar las diferencias entre las oportunidades de aprendizaje ofrecidas por una unidad de un texto escolar y las clases asociadas a este para desarrollar los contenidos y las habilidades científicas de acuerdo con el marco curricular chileno vigente en el 2009.

\section{Coherencia curricular: la necesidad de una visión comprensiva para la enseñanza de las Ciencias}

Dentro de las explicaciones desarrolladas para comprender cómo Finlandia logró los resultados en PISA 2006 en Ciencias, dos factores resultan significativos para este estudio. Por un parte, el énfasis en el desarrollo de las competencias científicas alineadas con aquellas medidas por la OCDE y, por otra, un trabajo sistemático para lograr en el sistema educativo una visión comprensiva y consistente de la enseñanza de las Ciencias (HAUTAMÄKI et al., 2008; LAVONEN; LAAKSONEN, 2009). Otro antecedente relevante es el estudio realizado por Valverde y Schmidt (1998) sobre los bajos resultados obtenidos por los estudiantes estadounidenses en la primera versión de TIMSS. Se concluyó que la reforma educativa había sido un proceso fragmentario y disperso, focalizado principalmente en la cobertura curricular por sobre el desarrollo de las habilidades científicas. De igual modo, Liu (2009) señala que el alineamiento curricular es una condición necesaria para lograr una visión integrada de lo que debe ser enseñado y evaluado en el campo de las Ciencias. En este sentido, el alineamiento curricular es un principio central en el contexto de una reforma educativa en cualquier campo disciplinar (BHOLA; IMPARA; BUCKENDAHL, 2003; HERMAN; WEBB; ZUNIGA, 2007; LIU, 2009).

En este trabajo se entenderá por alineamiento, a la relación existente entre lo declarado en cuanto a contenidos y habilidades por alcanzar en el currículo prescrito y lo efectivamente medido por el sistema de evaluación a gran escala, desarrollado por un determinado sistema educativo. Por lo tanto, el alineamiento curricular es una propiedad que opera a nivel de política educativa y del macro-sistema escolar. Tal como plantean Bhola, Impara y Buckendahl (2003), el concepto de alineamiento no es nuevo en el campo de las evaluaciones a gran escala; sin embargo, es relativamente novedosa la relación entre estándares de contenido y evaluación de los mismos como medida y palanca de cambio en contextos de reforma curricular.

Sin embargo, el estudio de Penuel et al. (2009) muestra que el alineamiento curricular no es el único principio para poder alcanzar éxito en un proceso de reforma educativa. Se debe, asimismo, considerar la implementación curricular, que contempla la elaboración de materiales y el desarrollo profesional de los docentes. Por lo tanto, es fundamental no solo establecer vínculos entre los componentes del macro sistema, sino también entre este y el microsistema. Porter et al. 
(2007) establecieron que la medida de alineamiento no solo debe ser considerada entre currículo y evaluación, sino también en relación con los textos escolares y las prácticas educativas. Porter et al. (2007) utilizan un índice para considerar el efecto de la variable profesor en el éxito de una implementación curricular. A través de matrices de contenido establecen los énfasis dados por los profesores en su enseñanza dentro de las aulas. De este modo, plantean una medida interesante para establecer relaciones entre profesores, currículo, logros de los estudiantes y materiales curriculares. En este trabajo, se operacionalizó como coherencia curricular - según el Committee on Science Education K-12 and the Mathematical Sciences Education Board (1999) - el grado de conexión y desarrollo de contenidos nucleares y habilidades a través de las diferentes unidades didácticas, de los textos escolares y de las clases realizadas.

Con el fin de poder medir el grado de coherencia con que son presentados, evaluados e implementados los contenidos y las habilidades científicas en el sistema educativo chileno, se han determinado distintos niveles de concreción curricular como lo muestra la Figura 1.

Figura 1. Niveles de concreción curricular

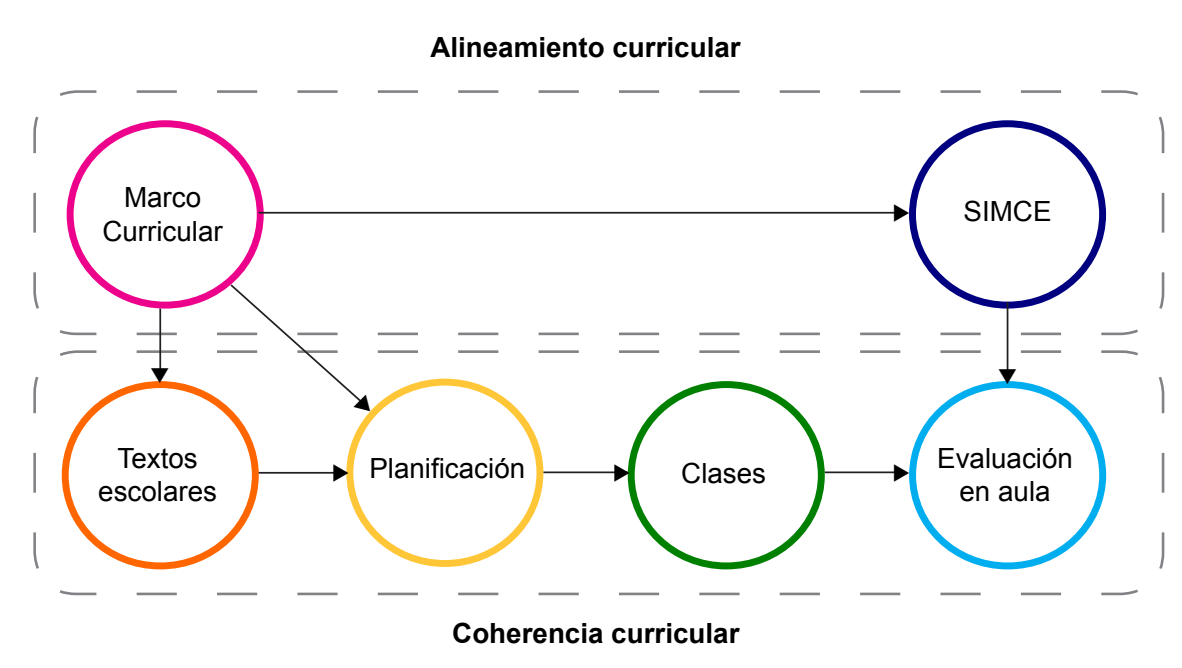

Fuente: elaborada por los autores.

Para establecer los contenidos y habilidades científicas que son promovidas en las prácticas de aula, en contraste con las enfatizadas en el texto escolar y con las declaradas por el marco curricular, es necesario establecer un punto de articulación entre distintos niveles de concreción curricular, cuyas condiciones de producción y circulación son distintas. McDonnell (1995) demuestra las ventajas de operar con el concepto de oportunidad de aprendizaje no solo en el campo de la evaluación estandarizada, sino también en el de la investigación educativa, porque no puede ser evaluado aquello que no ha sido enseñado. Este concepto permite comparar los grados de coherencia entre el currículo prescrito, el implementado y el evaluado, al definir una unidad de medida común entre distintos niveles (ANDERSON, 2002; McDONNELL, 1995; 
PORTER, 2009). Para este estudio, se operacionalizó la noción de oportunidad de aprendizaje de una habilidad en un contenido, así como la presencia de una actividad pedagógica que promueve dicha habilidad en relación con el contenido.

Las preguntas que orientan este estudio son: ¿Qué habilidades científicas de acuerdo al currículo chileno 2009 son promovidas por el texto escolar de $5^{\circ}$ grado para el contenido "Fuerza"?; ¿Qué habilidades científicas de acuerdo al currículo chileno 2009 son fomentadas en las actividades de clase de $5^{\circ}$ para dicho contenido?; ¿Cuál es el grado de coherencia curricular entre el marco curricular y el texto escolar para el contenido "Fuerza" en $5^{\circ}$ grado?; ¿Cuál es el grado de coherencia entre el texto escolar y las clases realizadas para dicho contenido? y; ¿entre el marco curricular y las actividades de aula para ese contenido?

\section{Metodología}

Este estudio descriptivo exploratorio buscó caracterizar las habilidades científicas promovidas en los textos escolares y aquellas desarrolladas en el aula para el contenido "Fuerza" correspondiente a $5^{\circ}$ grado; también determinar el grado de coherencia entre las oportunidades de aprendizaje declaradas en el marco curricular, las promovidas en un texto escolar y en la clase de Ciencias. Específicamente, se pretendió aplicar el índice de Porter et al. (2007) en diferentes niveles de la concreción curricular, mostrando el potencial innovador de este índice para ayudar a la política pública en la toma decisiones y a los docentes en el uso efectivo de los materiales curriculares.

\section{Muestra}

Se seleccionó un corpus de datos recolectados etnográficamente durante el año 2005 por Meneses (2008). Se trabajó con los datos de $5^{\circ}$ grado del sector de Ciencias, recolectados en un colegio particular de financiamiento compartido ubicado en la Región Metropolitana, Chile.

\section{Estrategias de recolección de la información}

La subunidad seleccionada corresponde al subsector de Física, al eje "Fuerza y Movimiento". Las técnicas utilizadas fueron:

1. Análisis de documentos: se empleó el marco curricular nacional (CHILE, 2002) y el texto escolar (DÍAZ; CIFUENTES, 2004) licitado por el Ministerio de Educación de Chile y utilizado en el aula para la enseñanza de las Ciencias en el período de la etnografía;

2. Observación no participante: se utilizó dicha técnica para la recolección de información sobre actividades de aula. Se seleccionaron dos clases de 52 y 38 minutos respectivamente; ambas clases fueron video grabadas. Los contenidos de "Fuerza sobre los cuerpos" fueron cubiertos en las dos clases analizadas. 


\section{Estrategias de análisis de la información}

Para medir la coherencia curricular, se utilizó una estrategia cualitativa-cuantitativa en dos fases:

- Fase 1, cualitativa: los discursos recolectados (marco curricular, texto escolar y clases) fueron analizados según principios del análisis del discurso (ARNOUX, 2006; CHARAUDEAU; MAINGUENEAU, 2005). Los estudios sobre oportunidades de aprendizaje (McDONNELL, 1995) utilizan como principal instrumento el cuestionario a profesores, acercándose a las prácticas educativas indirectamente a través de lo declarado por estos. Sin embargo, el análisis del discurso ofrece la posibilidad de trabajar directamente con los discursos de cada nivel de concreción curricular. Las categorías utilizadas para la medición de las oportunidades de aprendizaje correspondierón a los contenidos y habilidades declarados por el marco curricular chileno para Ciencias, actualización 2002.

El texto escolar fue segmentado espacialmente en cada página según los propósitos pedagógicos de la información en ella (definición, diagrama, micro actividad, experimento, entre otros) y la extensión de cada elemento fue medida en base al área absoluta cubierta por el segmento. Las clases, a su vez, fueron segmentadas temporalmente (1 minuto utilizando Videograph). (RIMMELE, 2009). El foco de análisis fue la actividad realizada por profesor y estudiantes y no las interacciones verbales. En cada uno de los segmentos medidos, se determinó la presencia/no presencia de las categorías señaladas.

- Fase 2, cuantitativa: se construyeron tablas de contingencia para las variables categóricas (contenidos y habilidades) de cada segmento temporal y espacial identificadas en la fase cualitativa. Para ello, se «contó» el número de segmentos medidos que poseían un contenido y una habilidad. Se normalizó la tabla de contingencia dividiendo cada elemento por el número total de segmentos medidos en ese nivel de concreción curricular. Así se obtuvo la matriz de contenidos $M(c, h)$ donde $c$ representa los contenidos y $h$ representa las habilidades científicas. El número $M(c, h)$ corresponde a la proporción de segmentos con el contenido $c$ y la habilidad h. Para comparar las dos matrices de contenidos para cada nivel de concreción curricular (texto escolar y clases) se aplicó el índice de Porter et al. (2007), que calcula la diferencia entre la proporción medida entre contenidos y habilidades. Para calcular el índice de coherencia se utilizó la siguiente expresión:

$$
\text { Índice de coherencia }=1-\frac{1}{2} \sum_{\mathrm{c} ; \mathrm{h}} \mid \mathrm{M}_{1}(\mathrm{c} ; \mathrm{h})-\mathrm{M}_{2}(\mathrm{c} ; \mathrm{h})
$$

Donde $M_{1}(c ; b)$ corresponde al elemento asociado al nivel de concreción curricular 1 , relacionado con el contenido $c$ y con la habilidad $h$. De igual modo, $M_{2}(c ; b)$ corresponde al elemento relacionado con el nivel de concreción curricular 2 vinculado con el mismo contenido $c$ y con la misma habilidad $b ;|x|$ es el valor absoluto de $x$, y la suma $\sum$ se efectúa sobre todos los elementos de la matriz. En la expresión, el índice se calcula sumando el valor absoluto de la diferencia entre los 2 elementos correspondientes de cada matriz y luego, el resultado de la suma se divide por 2 y se resta a 1 . El índice varía entre 0 y 1 , siendo 0 ausencia de coherencia y 1 coherencia máxima: en el caso de coherencia máxima, los elementos de ambas matrices son idénticos, su diferencia es cero y se obtiene 1; en el caso de coherencia mínima, cada elemento que es 0 en una matriz es 1 en la otra y, por lo tanto, la suma de los valores absolutos da 2, que al dividir por 2 cancela completamente el 1 inicial. 


\section{Contenidos y habilidades científicas declarados en el currículum nacional chileno}

El Ministerio de Educación de Chile define en el Marco Curricular los contenidos mínimos y objetivos fundamentales que los establecimientos educativos chilenos deben cubrir durante el año escolar. El currículo vigente al momento de realizar las observaciones de clases y con el que se evaluó el texto utilizado es la actualización 2002. (CHILE, 2002). En él solo se determinan los contenidos mínimos que deben presentarse a los estudiantes durante la unidad "Fuerza y movimiento" (CHILE, 2002, p. 127): (1) Reconocer los tipos de trayectoria de un móvil; (2) Reconocer la forma de describir el movimiento; (3) Apreciar los efectos de una fuerza sobre los cuerpos y (4) Aplicación de las ideas de fuerza y peso a máquinas simples (palancas y balanza). A estos cuatro contenidos se agregó una quinta categoría - "No contenido" - para señalar todos aquellos contenidos revisados en la clase y en el texto y que no corresponden a los determinados por el Currículo o bien aquello destinado al manejo de grupo y a la gestión del clima de aula.

También, el Marco Curricular Chileno (CHILE, 2002, p. 125-126) especifica que los estudiantes deben desarrollar habilidades y actitudes propias del quehacer científico. La inclusión de habilidades científicas asociadas a contenidos mínimos obligatorios se acentúa en la “Actualización 2009” (CHILE, 2010). A diferencia del currículo 2002, el 2009 son incorporados como objetivos fundamentales las habilidades científicas.

El análisis del Marco Curricular (CHILE, 2002) permitió identificar ocho habilidades científicas: (1) formulación de preguntas, (2) elaboración de hipótesis, (3) análisis de hipótesis, (4) observación, (5) registro de datos, (6) ordenamiento de la información, (7) interpretación de información y (8) explicaciones. A estas ocho, se agregaron dos más que son explicitadas posteriormente (CHILE, 2009b) y que estaban presentes en el texto escolar analizado: descripción de datos y procedimientos. También se incluyó la categoría no babilidad para incluir otras actividades, por ejemplo, transcripción de información. Se emplearon las siguientes definiciones operacionales para las habilidades:

- Formulación de preguntas: elaboración de preguntas coherentes y pertinentes a la problemática.

- Elaboración de hipótesis: explícita afirmación de causalidad o no causalidad entre 2 o más eventos concurrentes (ZIMMERMAN, 2007).

- Análisis de hipótesis: determinación de la coherencia y pertinencia de una hipótesis en relación a la problemática.

- Observación: identificación de las características relevantes de un fenómeno.

- Registro de datos: uso de algún sistema de memoria externo para almacenar los datos.

- Explicaciones: en particular, Explicación científica. Uso evidencias científicas para relacionar causalmente un fenómeno natural o una experiencia.

- Ordenamiento de la información: identificación de información relevante y su organización comprensible en alguna forma de representación.

- Interpretación de información: conversión de información representada de una forma a otra. Uso de evidencia para apoyar argumentos o transformar evidencia presentada de alguna forma en argumentos. 
- Descripción de datos: identificación de las variables pertinentes a medir.

- Procedimientos: secuenciación coherente y lógica de los pasos para llevar a cabo adecuadamente una actividad.

- No habilidad: actividades destinadas a la trasmisión de información. Por ejemplo dictar, recordar, escribir pizarra, entre otros.

\section{Resultados}

\section{Oportunidades de desarrollo de habilidades científicas en el texto escolar}

De las unidades del texto escolar, fue seleccionada solamente la subunidad "Fuerza y Movimiento". Se presentan en la Tabla 1 una matriz de contenidos con las probabilidades obtenidas para las oportunidades de aprendizajes de los contenidos y las habilidades ofrecidas por la unidad "Fuerza y Movimiento" del texto escolar. La primera fila indica los contenidos mínimos obligatorios que deben estar presentes en la unidad; de los cuatro declarados en el currículo, solamente se indican que se identificaron dos: "Tipos de trayectorias de un móvil" y "Efectos de una fuerza sobre los cuerpos". La primera columna indica las habilidades asociadas a tales contenidos; las ocho primeras habilidades corresponden a aquellas oficialmente declaradas en el Marco Curricular, mientras que las tres restantes se incluyeron para fines del estudio. Los números de la segunda y tercera columna corresponden a la probabilidad medida para la oportunidad de aprendizaje de cada una de las habilidades en cada uno de los contenidos. La última columna presenta el porcentaje en que cada habilidad está presente en el texto, independiente del contenido, y la última fila indica el porcentaje en que cada contenido está presente, independiente de la habilidad desarrollada. Los números entre paréntesis indican un intervalo de confianza del $95 \%$ para las probabilidades y los porcentajes indicados.

Tabla 1. Matriz de contenidos para el texto escolar de Ciencias de 5 básico, unidad de "Fuerza y Movimiento"

\begin{tabular}{lrrrrrr}
\hline \multicolumn{1}{c}{ Habilidades } & \multicolumn{7}{c}{ Contenidos } \\
\cline { 2 - 7 } & $\begin{array}{c}\text { Tipos de trayectoria } \\
\text { de un móvil }\end{array}$ & $\begin{array}{c}\text { Efectos de una fuerza } \\
\text { sobre los cuerpos }\end{array}$ & $\begin{array}{r}\text { Porcentaje } \\
\text { habilidades }\end{array}$ \\
\hline Formulación de preguntas & 0.000 & $(0.000,0.000)$ & 0.000 & $(0.000,0.000)$ & 0.0 & $(0.0,0.0)$ \\
Elaboración de hipótesis & 0.000 & $(0.000,0.000)$ & 0.052 & $(0.047,0.058)$ & 5.2 & $(4.7,5.8)$ \\
Análisis de hipótesis & 0.000 & $(0.000,0.000)$ & 0.000 & $(0.000,0.000)$ & 0.0 & $(0.0,0.0)$ \\
Observación & 0.009 & $(0.007,0.012)$ & 0.233 & $(0.222,0.244)$ & 24.2 & $(23.1,25.3)$ \\
Registro de datos & 0.000 & $(0.000,0.000)$ & 0.106 & $(0.098,0.114)$ & 10.6 & $(9.8,11.4)$ \\
Ordenamiento de la información & 0.000 & $(0.000,0.000)$ & 0.010 & $(0.007,0.012)$ & 1.0 & $(0.7,1.2)$ \\
Interpretación de información & 0.000 & $(0.000,0.000)$ & 0.147 & $(0.138,0.157)$ & 14.7 & $(13.8,15.7)$ \\
Explicaciones & 0.000 & $(0.000,0.000)$ & 0.133 & $(0.124,0.142)$ & 13.3 & $(12.4,14.2)$ \\
Descripción de datos & 0.000 & $(0.000,0.000)$ & 0.000 & $(0.000,0.000)$ & 0.0 & $(0.0,0.0)$ \\
Procedimientos & 0.000 & $(0.000,0.000)$ & 0.125 & $(0.117,0.134)$ & 12.5 & $(11.7,13.4)$ \\
No habilidad & 0.045 & $(0.039,0.050)$ & 0.140 & $(0.131,0.149)$ & 18.5 & $(17.5,19.5)$ \\
Porcentaje contenidos & 5.4 & $(4.8,6.0)$ & 94.6 & $(94.0,95.2)$ & &
\end{tabular}

Fuente: elaborada por los autores. 
En la unidad del texto analizada, solo se cubren dos contenidos y la mayor proporción corresponde a "Fuerza sobre los cuerpos". Este contenido aborda un 95\% de la unidad. Del mismo modo, la habilidad más desarrollada en el texto escolar para la unidad es observación con $24.2(23.3,25.1) \%$; seguida por la no habilidad con $18.5(17.5,19.5) \%$. Esta última corresponde a aquella proporción del texto que entrega definiciones o instrucciones por seguir. Interpretación de información, explicaciones y procedimientos están presentes con una proporción similar con alrededor de un 13\%. Mientras que registro de datos y elaboración de hipótesis están en una proporción que difiere de las anteriores con un 11 y $5 \%$ respectivamente. La habilidad menos desarrollada de las presentes es ordenamiento de la información con $1.0(0.7,1.2) \%$. Las habilidades formulación de preguntas, análisis de hipótesis, descripción de datos están ausentes en la unidad analizada del texto escolar.

Este desequilibrio entre contenidos y habilidades presentes, también se manifiesta en las probabilidades de las oportunidades de aprendizajes de cada uno de ellos. Es así que la mayor oportunidad de aprendizaje ofrecida al estudiante es para el desarrollo de la observación dentro del contenido de "Efectos de una fuerza sobre los cuerpos" con un valor de $0.233(0.222,0.244)$. Este valor es significativamente mayor que el valor deseado de $1 / 16=0.0625$, que se obtiene al considerar que idealmente en cada uno de los 2 contenidos se debe desarrollar cada una de las 8 habilidades estipuladas, ofreciendo 16 oportunidades de aprendizaje diferentes. De hecho, de las diez oportunidades de aprendizaje presentes (aquellas con valores distintos de cero en la matriz de contenidos) solamente siete están relacionadas a las habilidades que el Marco Curricular declara como obligatorias, y de ellas, cuatro son superiores al valor ideal (observación, interpretación de información, explicaciones y registro de datos, todas para el contenido de "Efectos de una fuerza sobre los cuerpos"), mientras que las otras tres están bajo este valor ideal. Dentro del 5\% del texto que está dedicado a "Tipos de trayectorias de un móvil" la mayor probabilidad corresponde al desarrollo de la no habilidad, que en este caso corresponde a varios párrafos dedicados a definiciones de los diferentes tipos de trayectorias y a una micro actividad donde se pide al alumno que dibuje la trayectoria que cree que seguiría una pelota al ser lanzada y que compare su dibujo con algunos de sus compañeros.

Se puede concluir que, aunque el texto escolar ofrece una amplia variedad de actividades para el desarrollo de las habilidades científicas (7 de 10 habilidades), ellas se presentan mayoritariamente en relación con solo un contenido. Así, la unidad de texto no ofrece equidad en las oportunidades de aprendizaje para los contenidos y las habilidades.

\section{Oportunidades de desarrollo de habilidades científicas en actividades de clase}

La Tabla 2 muestra la matriz de contenidos obtenida para las dos clases. Se indica la probabilidad medida de las oportunidades de aprendizaje para los contenidos y las habilidades presentes en las actividades realizadas en las clases de Ciencias correspondientes a la unidad "Fuerza y Movimiento". Los números de la segunda y tercera columna se refieren a la probabilidad medida para la oportunidad de aprendizaje de cada habilidad en cada contenido. La última columna presenta el porcentaje en que cada habilidad está presente en el aula, independiente del contenido; y la última fila indica en qué porcentaje cada contenido está presente, independiente de la habilidad desarrollada. Los números entre paréntesis indican un intervalo de confianza del 95\% para las probabilidades y los porcentajes indicados. 
Tabla 2. Matriz de contenido para las actividades en dos clases de Ciencias de 5 básico, donde se abarcó la unidad de "Fuerza y Movimiento"

\begin{tabular}{lrrrrrr}
\hline \multicolumn{1}{c}{ Habilidades } & \multicolumn{7}{c}{ Contenidos } \\
\cline { 2 - 7 } & $\begin{array}{l}\text { Efectos de una fuerza } \\
\text { sobre los cuerpos }\end{array}$ & $\begin{array}{c}\text { No contenido unidad } \\
\text { de aprendizaje }\end{array}$ & $\begin{array}{r}\text { Porcentaje } \\
\text { habilidades }\end{array}$ \\
\hline Formulación de preguntas & 0.000 & $(0.000,0.000)$ & 0.000 & $(0.000,0.000)$ & 0.0 & $(0.0,0.0)$ \\
Elaboración de hipótesis & 0.024 & $(0.003,0.063)$ & 0.003 & $(0.000,0.019)$ & 2.7 & $(0.4,6.8)$ \\
Análisis de hipótesis & 0.013 & $(0.001,0.045)$ & 0.003 & $(0.000,0.018)$ & 1.6 & $(0.1,5.0)$ \\
Observación & 0.029 & $(0.005,0.072)$ & 0.003 & $(0.000,0.019)$ & 3.2 & $(0.7,7.7)$ \\
Registro de datos & 0.000 & $(0.000,0.000)$ & 0.000 & $(0.000,0.000)$ & 0.0 & $(0.0,0.0)$ \\
Ordenamiento de la información & 0.000 & $(0.000,0.000)$ & 0.000 & $(0.000,0.000)$ & 0.0 & $(0.0,0.0)$ \\
Interpretación de información & 0.000 & $(0.000,0.000)$ & 0.000 & $(0.000,0.000)$ & 0.0 & $(0.0,0.0)$ \\
Explicaciones & 0.205 & $(0.132,0.293)$ & 0.013 & $(0.001,0.044)$ & 21.9 & $(14.2,30.9)$ \\
Descripción de datos & 0.000 & $(0.000,0.000)$ & 0.000 & $(0.000,0.000)$ & 0.0 & $(0.0,0.0)$ \\
Procedimientos & 0.035 & $(0.008,0.080)$ & 0.003 & $(0.000,0.018)$ & 3.7 & $(0.9,8.4)$ \\
No habilidad & 0.504 & $(0.404,0.606)$ & 0.166 & $(0.098,0.247)$ & 66.9 & $(57.2,76.0)$ \\
Porcentaje contenidos & 81.0 & $(72.5,88.3)$ & 19.0 & $(11.7,27.5)$ & &
\end{tabular}

Fuente: elaborada por los autores.

El único contenido presente fue el asociado a "Efectos de una fuerza sobre los cuerpos" con un 81\% del tiempo; el resto del tiempo estuvo dedicado a actividades como gestión de aula o a contenidos no directamente relacionados con la actividad. Por ejemplo, la profesora condujo la conversación inicial sobre roce hacia la vestimenta de los deportistas a través del tiempo, sin retornar al concepto "roce".

En relación con las habilidades, en las clases predomina temporalmente la no habilidad, que en este caso correspondía a la repetición de los contenidos del texto de manera oral y escrita con un $66.8(58.6,74.4) \%$. La siguiente habilidad más desarrollada es explicaciones con 21.9 $(14.2,20.9) \%$. Las habilidades procedimientos, observación y elaboración de hipótesis están presentes con alrededor de 3\%, mientras que tienen menor presencia análisis de hipótesis con 1.6\% $(1.9,4.2) \%$. Las habilidades formulación de preguntas, registro de datos, ordenamiento de información, interpretación de información y descripción de datos están ausentes. Cabe destacar que de las ocho habilidades requeridas solamente cuatro están presentes en las clases analizadas.

En relación con las probabilidades de oportunidades de aprendizaje, se observa la misma tendencia que en el texto, donde solo algunas de las habilidades son desarrolladas y preferentemente vinculadas con solo un contenido. De hecho, a los estudiantes se les ofrece mayoritariamente la oportunidad de desarrollar la no habilidad en el único contenido de la clase, con una probabilidad un poco mayor a 1/2: más de la mitad de las oportunidades ofrecidas en las clases corresponde a la repetición pasiva de contenidos presentes en el texto. En segundo lugar, la habilidad explicaciones en el contenido "Efectos de una fuerza sobre cuerpos" tiene un valor de 0.205 , aproximadamente $1 / 5$. Las restantes probabilidades no superan el 0.03 , con valores que son significativamente menores al valor deseado de $0.0625(1 / 16)$, calculado en la sección anterior. Incluso algunas de las oportunidades ofrecidas corresponden a habilidades desarrolladas en el no contenido, como es el caso de la oportunidad para desarrollar explicaciones, con una probabilidad que no es significativamente diferente a la oportunidad para desarrollar análisis de hipótesis en el contenido "Efectos de una fuerza sobre los cuerpos". Estos valores bajo el ideal contrastan con los valores de las probabilidades de las oportunidades ofrecidas 
por el texto, donde por lo menos 5 oportunidades diferentes estaban sobre el valor ideal. Estas diferencias son un indicio de la baja coherencia entre las clases y el texto utilizado en ellas.

Por otro lado, se puede observar que las clases ofrecen una menor variedad de oportunidades para el desarrollo de habilidades científicas (5 de 10 habilidades) en comparación con las ofrecidas por el texto escolar para la misma unidad (7/10 habilidades); sin embargo, nuevamente ellas son desarrolladas solamente para un contenido.

\section{Grado de coherencia entre el marco curricular, el texto escolar y actividades de clases}

En el caso del texto, la matriz de contenido se presenta en la Tabla 1, mientras que para las clases analizadas se observa en la Tabla 2. El índice de coherencia obtenido entre el texto y la clases es $0.360(0.311,0.423)$. Recordando que el índice de coherencia varía entre 0 y 1 , siendo 0 coherencia nula y 1 , coherencia máxima, el valor obtenido muestra la baja coherencia existente entre el texto y las clases. El análisis de la matriz de contenido de cada uno de estos niveles de concreción curricular ya había mostrado una diferencia entre los énfasis desarrollados en el texto y aquellos desarrollados por la profesora en las clases. Aunque en la videograbación es patente el uso del texto en el aula, su uso se reduce mayoritariamente a leer, repetir y copiar las definiciones.

Para construir la matriz de contenidos para el Marco Curricular se decidió asignar a cada uno de los cuatro contenidos de la unidad de "Fuerza y Movimiento", la oportunidad de desarrollar las ocho habilidades declaradas en él, asignado una probabilidad de 1/32 a cada una de ellas. Esto se debe a que en el ajuste curricular de 2002 (CHILE, 2002), las habilidades no están claramente especificadas como en versiones posteriores. Las dos habilidades agregadas (descripción de datos y procedimientos) no fueron incluidas en la matriz del Marco Curricular y, por ende, en el cálculo del índice de coherencia. Al calcular el índice de coherencia entre el texto y el marco curricular, se obtuvo un valor de $0.267(0.105,0.409)$ que muestra una baja coherencia entre las oportunidades de aprendizaje propuestas por el Ministerio y aquellas ofrecidas por el texto. Esta baja coherencia señala que a pesar de las revisiones exhaustivas que sufren los textos licitados por el gobierno de Chile, esos textos no ofrecen equidad de oportunidades de aprendizajes a los estudiantes que los utilizan.

Por otro lado, al calcular el índice de coherencia entre el marco curricular y las clases se obtuvo $0.133(0.023,0.278)$, que es aún más bajo que el anterior. Podemos considerar este resultado como el efecto combinado de la baja coherencia del texto con el Marco Curricular y la baja coherencia entre el texto y la implementación en la clase: en las actividades observadas, se utilizan fragmentos del texto, el que ya posee un bajo índice de coherencia con el Marco Curricular. Con esto se reduce aún más la coherencia. Sin embargo, a partir de los intervalos de confianza, no se puede sostener que el índice entre las clases y el Marco Curricular sea significativamente menor que el índice entre el texto y el Marco Curricular, pero sí, que es significativamente menor al índice entre la clase y el texto.

La Figura 2 muestra los tres índices calculados entre los tres niveles de concreción curricular considerados en este estudio. Los números en cada flecha indican el índice de coherencia curricular entre los niveles conectados por éstas. El índice de coherencia curricular varía entre 0 y 1 , donde 0 indica coherencia mínima y 1, coherencia máxima. 
Figura 2. Índice de coherencia curricular para los tres niveles de concreción curricular analizados en el estudio

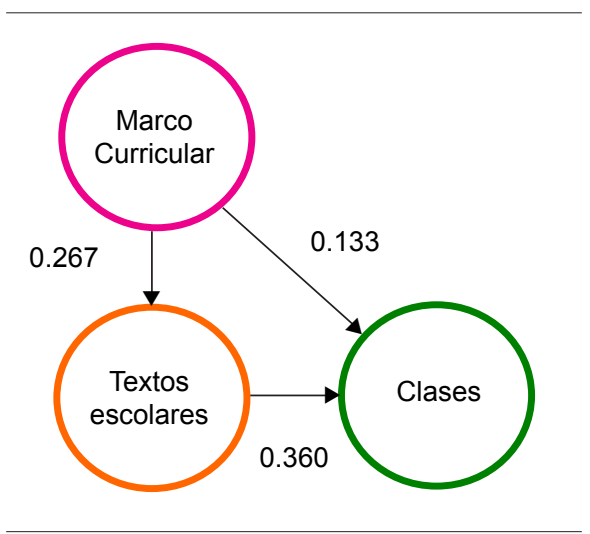

Fuente: elaborada por los autores.

\section{Conclusiones y proyecciones}

Este estudio muestra la viabilidad de aplicar el procedimiento desarrollado por Porter et al. (2007) para calcular el grado de coherencia entre las oportunidades de aprendizajes ofrecidas, promovidas e implementadas en distintos niveles de concreción curricular. Se aplicó este procedimiento para medir la coherencia entre los contenidos y habilidades declarados por el Marco Curricular chileno, las ofrecidas por el texto licitado y las proporcionadas en las actividades de clases para la enseñanza de las Ciencias, dentro de la unidad de "Fuerza y Movimiento" para el nivel de $5^{\circ}$ básico.

Un primer paso de este procedimiento consiste en construir una tabla, donde se presentan las probabilidades en que las oportunidades de aprendizajes se ofrecen para cada habilidad y contenido en los niveles curriculares estudiados. Un primer análisis de esta tabla permite determinar aquellos contenidos y habilidades que son mayoritariamente promovidas en cada nivel. Luego, al comparar las tablas de dos niveles de concreción curricular se obtiene el índice de coherencia entre ambos niveles. De este modo, este índice de coherencia permite detectar diferencias entre lo declarado y lo implementado en el sistema educativo.

Este trabajo muestra que a pesar de que el Marco Curricular (CHILE, 2002, 2010) declara específicamente los contenidos mínimos y habilidades científicas que deben ser desarrollados para este nivel educacional, las oportunidades ofrecidas dentro del texto escolar y a través de las actividades de clase no son proporcionales, favoreciendo algunas sobre otras. En efecto, para el caso del texto (Tabla 1), se encontró que entre los dos contenidos de la unidad, uno de ellos está presente en una proporción cercana al 95\%. Similarmente, se detectó que de las 10 habilidades científicas declaradas por el Ministerio, solamente 7 están presentes, y de esas, 5 corresponden casi al 75\%. También se encontró que una proporción no menor, un 18\% del texto, promueve la no habilidad, reduciendo el aprendizaje a la mera repetición de información.

Este desequilibrio en la promoción de habilidades y contenidos tiene un impacto directo en el desarrollo de habilidades y el aprendizaje de los contenidos de los estudiantes que utilizan 
este texto. Esto llama aún más la atención si se considera que el texto escolar analizado es parte de los recursos curriculares entregados por el gobierno de Chile al 95\% de los estudiantes del país. (CHILE, 2009a). Las consecuencias de esta desproporción se ven acentuadas al considerar el nivel aula. Como se observa en la Tabla 2, la habilidad más desarrollada, explicaciones, no corresponde con la que tiene más presencia en el texto, observación. Además, alrededor de un 70\% del tiempo de las actividades de clase promueven la no-babilidad. Una limitación de este estudio es no contar con información sobre las decisiones pedagógicas que subyacen a las actividades observadas en el aula.

Por otro lado, se encontró que la menor coherencia corresponde a aquella entre el marco curricular y las clases $(0.133(0.031,0.256))$. Esta baja coherencia se puede explicar por el efecto combinado de la baja coherencia, por un lado, entre lo declarado por el marco curricular y lo ofrecido por el texto escolar y, por otro lado, por lo presentado en el texto escolar y lo implementado por el docente en el aula.

Una propuesta para aumentar la coherencia entre lo propuesto por el marco y lo realizado en las clases sería elaborar una política pública que asegurara la coherencia entre las oportunidades de aprendizaje promovidas en los textos escolares y lo establecido en los marcos curriculares.

Una proyección de este estudio sería determinar el índice de coherencia para otros niveles de concreción curricular, por ejemplo, la relación entre plan de clases y actividades observadas realizadas en el aula, entre actividades y evaluaciones de aula, entre otras.

Esta misma metodología de análisis podría ser utilizada para analizar el grado de coherencia entre oportunidades de aprendizaje ofrecidas también en otros sectores de aprendizaje. Asimismo, una extensión interesante consistiría en relacionar el grado de coherencia curricular con los aprendizajes de los estudiantes, con el fin de determinar si se trata de una condición necesaria para el aumento de su desempeño, o bien para contrastar las habilidades promovidas por el sistema educativo chileno con mediciones internacionales. Finalmente, este tipo de análisis podría ser utilizado para explicar las diferencias de desempeño entre distintos tipos de establecimientos escolares o perfiles docentes, entre otros.

En síntesis, la metodología presentada permite comparar efectivamente oportunidades de aprendizajes para detectar brechas en el sistema educativo. La información levantada a partir del análisis es de alta relevancia para la política educativa pues ofrece una visión amplia e inclusiva (macro y micro sistema) sobre la comprensión e implementación de una determinada reforma educativa. En efecto, oportunidad de aprendizaje e índice de coherencia curricular permiten identificar los niveles de implementación curricular donde las oportunidades de aprendizaje están siendo más débilmente promovidas para proponer soluciones concretas y de alto impacto tanto a nivel de política pública como a nivel escolar.

\section{Referencias}

ANDERSON, L. Curricular alignment: a re-examination. Theory into Practice, Philadelphia, v. 41, n. 4, p. 255-260, 2002.

ARNOUX, E. Análisis del discurso: modos de abordar materiales de archivo. Buenos Aires: Santiago Arcor, 2006. 
BHOLA, D. S.; IMPARA, J. C.; BUCKENDAHL, C. W. Aligning tests with states' content standards: methods and issues. Educational Measurement: issues and practice, Washington, v. 22, n. 3, p. 21-29, 2003.

CHARAUDEAU, P.; MAINGUENEAU, D. Diccionario de análisis del discurso. Madrid: Amorrortu, 2005.

CHILE. Ministerio de la Educación. Departamento de Estudios y Desarrollo. Estadísticas de la educación 2008. [S.1.], 2009a.

CHILE. Ministerio de Educación. Unidad de Currículum y Evaluación. Chile y el aprendizaje de matemáticas y ciencias según TIMSS. Santiago, 2004.

. Marco curricular de la educación básica: objetivos fundamentales y contenidos mínimos obligatorios de la educación básica. Actualización 2002. [S.1.], nov. 2002. Disponible en: <http:// curriculumenlinea.mineduc.cl/descargar.php? id doc=201102031002220 > . Acceso el: 01 mar. 2012.

Marco curricular de la educación básica: objetivos fundamentales y contenidos mínimos obligatorios de la educación básica. Actualización 2009. [S.1.], ene. 2010. Disponible en: <http:// curriculumenlinea.mineduc.cl/descargar.php? id doc=201203011128110 > . Acceso el: 01 mar. 2012.

Niveles de logro $4^{\circ}$ básico para comprensión del medio natural SIMCE. [S.l.], 2008.

2007.

. PISA 2006: rendimientos de estudiantes de 15 años en ciencias, lectura y matemáticas. [S.l.],

CHILE. Ministerio de Educación. Unidad de Currículum y Evaluación. ¿Qué nos dice PISA sobre la educación de los jóvenes en Chile? Santiago, 2009b.

COMMITTEE ON SCIENCE EDUCATION K-12 AND THE MATHEMATICAL SCIENCES EDUCATION BOARD. Designing mathematics or science curriculum programs: a guide for using mathematics and science education standards. Washington: National Academy Press, 1999.

DEBOER, G. E. Scientific literacy: another look at its historical and contemporary meanings and its relationship to science education reform. Journal of Research in Science Teaching, Hoboken, v. 37 , n. 6, p. 582-601, 2000.

DILLON, J. On scientific literacy and curriculum reform. International Journal of Environmental \& Science Education, Bolu, v. 4, n. 3, p. 201-213, 2009.

DÍAZ, A. S.; CIFUENTES, A. C. Estudio y comprensión de la naturaleza: $5^{\circ}$ básico. Santiago de Chile: Zig-Zag, 2004.

FENSHAM, P. J. De nouveaux guides pour l'alphabétisation scientifique. Canadian Journal of Science, Mathematics and Technology Education, Philadelphia, v. 2, n. 2, p. 133-149, 2002.

GUBLER, J.; WILLIAMSON, A. Resultados de los estudiantes chilenos en la prueba PISA ciencias 2006: una mirada a las competencias. In: CHILE. Ministerio de Educación. Unidad de Currículum y Evaluación. ¿Qué nos dice PISA sobre la educación de los jóvenes en Chile? Santiago, Chile: Ministerio de Educación, 2009. p. 197-237.

HAUTAMÄKI, J. et al. Pisa 2006 Finland: analyses, reflections, explanations. Helsinki: Ministry of Education, 2008. Disponible en: <http://www.pisa2006.helsinki.fi/files/PISA06_Analyses_ Reflections_and_Explanations.pdf $>$. Acceso el: 30 jul. 2014.

HERMAN, J. L.; WEBB, N. M.; ZUNIGA, S. A. Measurement issues in the alignment of standards and assessments. Applied Measurement in Education, Philadelphia , v. 20, n. 1, p. 101-126, 2007. 
HOLBROOK, J.; RANNIKMAE, M. The meaning of scientific literacy. International Journal of Environmental \& Science Education, Bolu, v. 4, n. 3, p. 275-288, 2009.

HURD, P. D. Scientific literacy: new minds for a changing world. Science Education, Hoboken, v. 82, n. 3, p. 407-416, 1998.

JENKINS, E. W. Reforming school science education: a commentary on selected reports and policy documents. Studies in Science Education, Abingdon, v. 45, n. 1, p. 65-92, 2009.

LAUGKSCH, R. C. Scientific literacy: a conceptual overview. Science Education, Hoboken, v. 84, n. 1, p. 71-94, 2000.

LAVONEN, J.; LAAKSONEN, S. Context of teaching and learning school science in Finland: reflections on PISA 2006 results. Journal of Research in Science Teaching, Hoboken, v. 46, n. 8, p. 922-944, 2009.

LIU XIUFENG. Beyond science Literacy: science and the public. International Journal of Environmental \& Science Education, Bolu, v. 4, n. 3, p. 301-311, 2009.

McDONNELL, L. M. Opportunity to learn as a research concept and policy instrument.

Educational Evaluation and Policy Analysis, Washington, v. 17, n. 3, p. 305-322, 1995.

MENESES, A. Leer y escribir en una escuela chilena: representaciones discursivas de los diferentes agentes educativos en las áreas prioritarias del currículo escolar en NB3. Revista Signos: estudios de linguística, Valparaiso, v. 41, n. 67, p. 257-278, 2008.

MILLAR, R. Twenty first century science: Insights from the design and implementation of a scientific literacy approach in school science. International Journal of Science Education, London, v. 28, n. 13, p. 1499-1521, 2006.

PENUEL, W. et al. Is alignment enough?: investigating the effects of state policies and professional development on science curriculum implementation. Science Education, Hoboken, v. 93, n. 4, p. 656-677, 2009. Disponible en: <http://dx.doi.org/ 10.1002/sce.20321>. Acceso el: 01 mar. 2012.

PORTER, A. C. The uses and misuses of opportunity to learn standards. Educational Researcher, Thousand Oaks, v. 24, n. 1, p. 21-27, 2009.

PORTER, A. C. et al. Alignment as a teacher variable. Applied Measurement in Education, Philadelphia, v. 20, n. 1, p. 27-51, 2007.

RIMMELE, R. Videograph: version 4.2.1.1. Kiel, Alemania: [s.n.], 2009. [Programa computacional].

ROBERTS, D. A. Scientific literacy / science literacy. In: ABELL, S. K.; LEDERMAN, N. G. (Ed.).

Handbook of research on science education. London: Lawrence Erlbaum, 2007. p. 729-780.

VALVERDE, G.; SCHMIDT, W. H. Refocusing U. S. math and science education. Issues in Science and Technology, Richardson, v. 14, n. 2, p. 60-66, 1998.

ZIMMERMAN, C. The development of scientific thinking skills in elementary and middle school.

Developmental Review, Maryland Heights, v. 27, n. 2, p. 172-223, 2007.

Artigo recebido em 09/03/12. Aceito em 15/06/13. 